\title{
Pure laparoscopic versus open right hepatectomy in living liver donors: which is longer bench-surgery time
}

\author{
Kwangpyo Hong, Suk Kyun Hong, Eui Soo Han, Sanggyun Suh, Su young Hong, Jeong-Moo Lee, YoungRok Choi, \\ Nam-Joon Yi, Kwang-Woong Lee, Kyung-Suk Suh
}

Division of Hepatobiliary, Department of Surgery, Seoul National University College of Medicine, Korea

Background: The aim of the study was to measure bench-surgery time in pure laparoscopic donor right hepatectomy (PLDRH) in comparison with those of conventional donor right hepatectomy (CDRH).

Methods: We retrospectively reviewed the medical records of 514 donors who underwent living donor liver transplantation between January 2012 and December 2019 at Seoul National University Hospital. We divided it into two periods: when almost only $\mathrm{CDRH}$ was performed and the second period during which PLDRH was standardized.

Results: The mean bench-surgery time (49.3 vs. 39.5 minutes; $P=0.00$ ) was longer in the PLDRH group than the CDRH group. We performed the analysis while excluding the factors affecting the bench-surgery time in both groups. First, we analyzed except the patients who had undergone direct venoplasty or Y-graft patch reconstruction of two portal veins. After that, we excluded the patients who had reconstruction of more than three openings of middle hepatic vein tributaries and no reconstruction of those (right hemi-liver graft). Lastly, we excluded the patients who underwent venoplasty of inferior hepatic vein(s) in back-table procedures. In all three subgroup analyses, the mean bench-surgery time was also longer in the PLDRH group than the CDRH group (47.4 vs. 38.4 minutes, $P=0.00 ; 48.7$ vs. 37.4 minutes, $P=0.00 ; 48.3$ vs. 36.6 minutes, $P=0.00$ ).

Conclusions: The bench-surgery time takes longer in the PLDRH group regardless of reconstruction of type II or III portal vein variations and liver graft outflow or not.

Corresponding author: Suk Kyun Hong

E-mail: nobel1210@naver.com

(c) The Korean Society for Transplantation

This is an Open Access article distributed under the terms of the Creative Commons Attribution Non-Commercial License (http://creativecommons.org/licenses/by-nc/4.0/) which permits unrestricted non-commercial use, distribution, and reproduction in any medium, provided the original work is properly cited. 